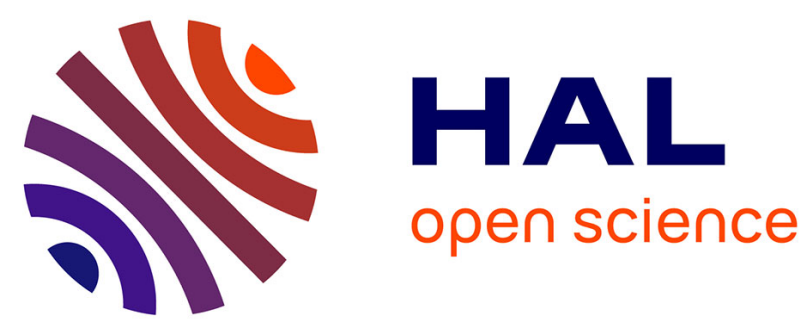

\title{
Extended Game-Theoretical Semantics
}

Manuel Rebuschi

\section{To cite this version:}

Manuel Rebuschi. Extended Game-Theoretical Semantics. Between Logic and Reality. Modeling Inference, Action and Understanding, 25, Springer, pp.161-182, 2012, Logic, Epistemology, and the Unity of Science, 978-94-007-2389-4. 10.1007/978-94-007-2390-0_9 . halshs-01231961

\section{HAL Id: halshs-01231961 https://shs.hal.science/halshs-01231961}

Submitted on 21 Nov 2015

HAL is a multi-disciplinary open access archive for the deposit and dissemination of scientific research documents, whether they are published or not. The documents may come from teaching and research institutions in France or abroad, or from public or private research centers.
L'archive ouverte pluridisciplinaire HAL, est destinée au dépôt et à la diffusion de documents scientifiques de niveau recherche, publiés ou non, émanant des établissements d'enseignement et de recherche français ou étrangers, des laboratoires publics ou privés.

\section{(이) $\$$}

Distributed under a Creative Commons Attribution - NonCommercial - NoDerivatives 44.0 


\title{
Extended Game-Theoretical Semantics
}

\author{
Manuel Rebuschi \\ L.H.S.P - Poincaré Archives \\ Nancy University (France) \\ manuel.rebuschi@univ-nancy 2. fr
}

July 4, 2010

\begin{abstract}
A new version of Game-Theoretical Semantics (GTS) is put forward where game rules are extended to the non-logical constants of sentences. The resulting theory, together with a refinement of our criteria of identity for functions, provide the technical basis for a game-based conception of linguistic meaning and interpretation.
\end{abstract}

Keywords: Game semantics, NL semantics, Meaning and interpretation, Skolem functions.

\section{Contents}

1 Introduction $\quad 2$

2 Game-Theoretical Semantics and Skolemization $\quad 3$

2.1 Usual GTS in a nutshell . . . . . . . . . . . . . . . . . . . 3

2.2 Extending GTS to non-logical constants . . . . . . . . . . . . . . 5

3 An Account of Meaning and Interpretation Based on Game Strategies 8

3.1 Meaning: Implemented Skolem functions . . . . . . . . . . . . . . . 8

3.2 Interpretation: Implemented Dig functions . . . . . . . . . . . . . . . 10

4 Application and Philosophical Consequences 12

4.1 Contrasting meaning and interpretation . . . . . . . . . . . . . 12

4.2 Philosophical puzzles . . . . . . . . . . . . . . . . 17

5 Conclusion $\quad 19$

Appendices $\quad 20$

A eGTS rules $\quad 20$

B Second-order Skolem forms $\quad 21$ 


\section{Introduction}

Game-theoretical semantics (hereafter, GTS) was created by Hintikka during the 1960s and intended as a formalization of Wittgenstein's remarks on language games. Like other formal connections drawn between logic and game theory, it embodies several philosophically relevant features into semantics: the dynamics of language, language as an activity, language as a collective matter. A specific insight is offered by GTS through the notion of strategic meaning. I will propose to use this notion in order to account for linguistic interpretation, to be contrasted with meaning constitution. It will yield solutions to several issues every theory of linguistic meaning is confronted with, especially those involving an epistemic dimension.

My proposal will be based on two moves: (1) the extension of semantic games to the subatomic components of sentences, and (2) a shift in our usual criteria of individuation of functions. The resulting conception of meaning is a two-component theory, but it does not use entities such as Frege's senses or Montague's functions defined on possible worlds. The main idea can be summed up as follows: in addition to the truth-conditions of a sentence, which are given by the existence of a winning strategy for the initial verifier of some semantic game, one should also consider the particular (implemented) strategies employed by concrete speakers. Such strategies correlated with the non-logical expressions of a given natural language consist in functions that pick up their extension relative to a given context. ${ }^{1}$ However, they need not realize winning strategies in every context, what is required being that they reach socially acceptable approximations of the extension at stake. Sets of such functions are what is mastered by the agents to understand linguistic expressions. This informal account of truth-conditional meaning and strategy-based interpretation will get a more precise working out within the framework of GTS.

In the next section I will briefly sketch the required technical apparatus: standard and extended GTS for first-order formal languages, and the conception of Skolem functions as components of winning strategies. In Section 3 I will look into the application of GTS to natural language, and deal with the question of how to use and individuate Skolem functions in extended GTS. Two notions will be defined: that of eGTS meaning, as a set of implemented Skolem functions, and that of eGTS stereotype (more or less regimenting Putnam's idea of idiolectal meaning) as a set of implemented (but not always winning) strategies. Next, Section 4 will be devoted to examining a few puzzles of the philosophy of language.

\footnotetext{
${ }^{1}$ Like Fodor's [4] narrow content. See [14] for an elaboration of the connexion between narrow content and the conception developed in the present paper.
} 


\section{Game-Theoretical Semantics and Skolemization}

\subsection{Usual GTS in a nutshell}

Semantic games The general idea of $\mathrm{GTS}^{2}$ is to associate to each firstorder (FO) sentence $\varphi$ evaluated relative to some structure $\mathbf{M}=\langle D, I\rangle$ a semantic game, denoted by $G(\varphi, \mathbf{M})$ and played between two abstract players, the initial verifier $\exists$ loise and the initial falsifier $\forall$ belard, s.t. the first player (resp. the second one) has a uniform winning strategy iff the formula is true (resp. false) in M. Such evaluation games are played according to the following rules:

- (R.At). If $\alpha$ is a true atomic sentence (or identity) in $\mathbf{M}$, then the verifier wins $G(\alpha, \mathbf{M})$, and the falsifier loses. If $\alpha$ is a false atomic sentence (or identity), the reverse obtains.

- (R. $\vee)$ In the game $G\left(\varphi_{1} \vee \varphi_{2}, \mathbf{M}\right)$ the verifier picks out an index $i \in\{1,2\}$. The rest of the game is as in $G\left(\varphi_{i}, \mathbf{M}\right)$.

- (R. $\wedge)$ In the game $G\left(\varphi_{1} \wedge \varphi_{2}, \mathbf{M}\right)$ the falsifier picks out an index $i \in\{1,2\}$. The rest of the game is as in $G\left(\varphi_{i}, \mathbf{M}\right)$.

- (R. $\exists)$ The game $G(\exists x \varphi(x), \mathbf{M})$ starts with the verifier choosing a member $d \in D$ and a new name $\mathbf{c}$ to designate it; the rest of the game is as in $G(\varphi(\mathbf{c}), \mathbf{M})$.

- (R. $\forall)$ The game $G(\forall x \varphi(x), \mathbf{M})$ is similar, except that the choice is made by the falsifier.

- (R. $\neg)$ The game $G(\neg \varphi, \mathbf{M})$ is like $G(\varphi, \mathbf{M})$, except that the roles of the two players (as defined by the rules) are interchanged.

With such rules, one can define truth and falsity according to GTS:

$\mathbf{M} \vDash_{\text {GTS }}^{+} \varphi(\varphi$ is GTS-true in $\mathbf{M})$ iff there is a winning strategy for the initial verifier ( $\exists$ loise $)$ in $G(\varphi, \mathbf{M})$.

$\mathbf{M} \vDash_{\mathrm{GTS}}^{-} \varphi(\varphi$ is GTS-false in $\mathbf{M})$ iff there is a winning strategy for the initial falsifier ( $\forall$ belard) in $G(\varphi, \mathbf{M})$.

Skolemization Assertion of the GTS truth-conditions for a sentence, i.e. of the existence of a winning strategy for $\exists$ loise in the associated game, is expressible in the $\Sigma_{1}^{1}$ fragment of second-order logic. Each strategy can be represented by a finite sequence of Skolem functions corresponding to the moves made by the verifier (rules (R. $\exists$ ) and (R.V)) relative to those played by the falsifier. This will yield e.g.:

$$
\begin{aligned}
& \mathbf{M} \vDash_{\text {GTS }}^{+} \forall x \exists y R(x, y) \Longleftrightarrow \mathbf{M} \vDash \exists f \forall x R(x, f(x)) \\
& \mathbf{M} \vDash_{\text {GTS }}^{+} \forall x\left(\varphi_{1} \vee \varphi_{2}\right) \Longleftrightarrow \mathbf{M} \vDash \exists f \forall x\left(\left(f(x)=0 \wedge \varphi_{1}\right) \vee\left(f(x) \neq 0 \wedge \varphi_{2}\right)\right)
\end{aligned}
$$

\footnotetext{
${ }^{2}$ See [9] for an overview.
} 
(In the second example one assumes that the model contains at least two elements, and that one of them is denoted by ' 0 '.)

Skolemization is in fact a well-known transformation of FO formulas: In a formula in prenex normal form, replace each existential quantifier $\exists x_{i}$ by a (new) function symbol $\mathbf{f}_{i}$ whose variables are those bound by the universal quantifiers of which $\exists x_{i}$ is within the scope. Following Hintikka, extend the introduction of function symbols to disjunctions (for models with at least two elements.) In what follows, $\mathbf{S k}[\varphi]$ will denote a formula resulting from $\varphi$ through such a transformation: ${ }^{3}$

$$
\begin{aligned}
\operatorname{Sk}[\forall x \exists y R(x, y)] & =\forall x R(x, \mathbf{f}(x)) \\
\operatorname{Sk}[\forall x \exists y \forall z \exists u \varphi(x, y, z, u)] & =\forall x \forall z \varphi\left[x, \mathbf{f}_{1}(x), z, \mathbf{f}_{2}(x, z)\right] \\
\operatorname{Sk}\left[\forall x\left(\varphi_{1} \vee \varphi_{2}\right)\right] & =\forall x\left(\left(\mathbf{f}(x)=0 \wedge \varphi_{1}\right) \vee\left(\mathbf{f}(x) \neq 0 \wedge \varphi_{2}\right)\right)
\end{aligned}
$$

A second-order Skolem form of a given formula $\varphi$, hereafter symbolized by 2Sk $[\varphi]$, obtains from a (first-order) Skolem form of $\varphi$ in which each Skolem function symbol is replaced by an existentially quantified functional variable:

$$
\mathbf{2 S k}[\forall x \exists y R(x, y)]=\exists f \forall x R(x, f(x))
$$

Assuming the Axiom of Choice and the standard "full" semantics of secondorder logic, $\varphi$ and $\mathbf{S k}[\varphi]$ are equisatisfiable, and $\varphi$ and $\mathbf{2} \mathbf{S k}[\varphi]$ are equivalent.

Truth-Conditions Assuming the Axiom of choice and the standard interpretation of second-order logic, the GTS truth-definition is equivalent to the standard one:

$$
\mathbf{M} \vDash_{\text {GTS }}^{+} \varphi \Longleftrightarrow \mathbf{M} \vDash \varphi .
$$

The equivalence between GTS and Tarski-like semantics leads to the inescapable fact that for any FO sentence there is always a winning strategy for one of the players: bivalence corresponds to the determination of the semantic games.

GTS provides a nice and intuitive interpretation of Skolemization: the Skolem functions occurring in $\mathbf{S k}[\varphi]$ are conceived of as the components of $\exists$ loise's winning strategies in $G(\varphi, \mathbf{M})$. For example in $\mathbf{M}=\langle D, I\rangle$, where $D=\mathbb{N}$ and " $<$ " gets its usual interpretation, the formula $\varphi=\forall x \exists y(x<y)$ is obviously true. It admits of one skolemization, $\mathbf{S k}[\varphi]=\forall x(x<\mathbf{f}(x))$, where the newly introduced symbol $\mathbf{f}$ can receive several values, each of which corresponds to a winning strategy available to $\exists$ loise: $\mathbf{f}_{1}(x)=x+1$, $\mathbf{f}_{2}(x)=2 x+1$, etc. The GTS-truth-conditions of $\varphi$ are given by $2 \mathbf{S k}[\varphi]=$ $\exists f \forall x(x<f(x))$.

\footnotetext{
${ }^{3}$ Of course, starting with a given FO formula there is no unicity in the upshot in the general case since several prenex normal forms can sometimes be available. This is the case with $\exists x A x \rightarrow \exists y B y$ which leads us to two prenex forms, $\forall x \exists y(A x \rightarrow B y)$ and $\exists y \forall x(A x \rightarrow B y)$, thus to two distinct skolemizations: respectively $\forall x(\neg A x \vee B \mathbf{f}(x))$ and $\forall x(\neg A x \vee B \mathbf{a})$, where $\mathbf{a}$ is a constant (function) symbol.
} 


\subsection{Extending GTS to non-logical constants}

In standard GTS, Skolem functions replace existential quantifiers and disjunctions. From a meta-semantic point of view, they correspond to what is taken into account by the assignments in Tarski-like semantics. What I will put forward now is extending such a role of Skolem functions to what is dealt with by the interpretation function $I$ of the model-theoretic structure $\mathbf{M}=\langle D, I\rangle$. To put it in another perspective: whereas GTS game rules are associated with logical constants, extended GTS (eGTS hereafter) will associate new rules with the non-logical vocabulary. ${ }^{4}$ The notion of semantic game is thus extended to atomic sentences (and identities).

Atomic games In eGTS, every sentence $\varphi$ evaluated relative to a structure $\mathbf{M}$ is associated to a game $e G(\varphi, \mathbf{M})$. This new game is identical to the game $G(\varphi, \mathbf{M})$ played according to the standard GTS rules when it is a molecular game, i.e. when $\varphi$ is a complex formula. If $\varphi$ is an atomic formula or an identity, one reaches an atomic game. The original game rule for atomic sentences (R.At) must then be replaced by four new rules (see Appendix A.)

First of all, if either $\exists$ loise is the current verifier and the atomic sentence is false, or she is the current falsifier and the sentence is true, then $\forall$ belard wins and $\exists$ loise loses. If it is not the case, then $\forall$ belard chooses one of its non-logical constants, an individual constant $a_{i}$, a function symbol $f_{j}$ or a relation symbol $R_{k}$, and the game goes on. ${ }^{5}$ When $\forall$ belard chooses an individual constant $a_{i}$, $\exists$ loise has to pick out an object in the domain; if she has selected the right value, i.e. the very extension of $a_{i}$, then she wins the play and $\forall$ belard loses. When $\forall$ belard chooses a $n$-ary function symbol $f_{j}$, he furthermore picks out a $n$-tuple of objects in the domain and $\exists$ loise must select an object in the domain: if it is identical to the value of $f_{j}$ for the given tuple then $\exists$ loise wins and $\forall$ belard loses. When $\forall$ belard chooses a $n$-ary relation symbol $R_{k}$, he furthermore picks out a $n$-tuple of objects in the domain and $\exists$ loise must check whether $R_{k}$ applies to this tuple; if $\exists$ loise succeeds, i.e. if she answers "yes" when $R_{k}$ applies to the tuple and "no" when it doesn't, then she wins the play and $\forall$ belard loses.

\footnotetext{
${ }^{4}$ Such an extension is actually suggested by Hintikka himself, see [7, p. 51]. However, this suggestion is essentially connected with applications in first-order epistemic logic, and no extended version of GTS is provided. Already in 1985 Hintikka and Kulas [8] argued that game rules must be associated with NL individual constants.

5 When an atomic game is reached there is no residual individual variable in the formula: as eGTS games are played for sentences only, every variable is bound and next replaced by a new constant during the molecular game. GTS could of course be defined for open formulas with a slight complication - games relative to a formula, a model and an assignement. However it is not necessary for the objectives of this paper.
} 
In eGTS, we thus have new truth and falsity definitions relative to a structure $\mathbf{M}=\langle D, I\rangle$ :

$\mathbf{M} \vDash_{\mathrm{eGTS}}^{+} \varphi(\varphi$ is eGTS-true in $\mathbf{M})$ iff there is a winning strategy for the initial verifier $(\exists$ loise $)$ in $e G(\varphi, \mathbf{M})$ played according to the eGTS-rules.

$\mathbf{M} \vDash_{\text {eGTS }}^{-} \varphi(\varphi$ is eGTS-false in $\mathbf{M})$ iff there is a winning strategy for the initial falsifier $(\forall$ belard) in $e G(\varphi, \mathbf{M})$ played according to the eGTS-rules.

It is worth noticing that according to the eGTS game rules, only individual objects are handled by the players - i.e., no higher-order entity is involved.

Extending Skolemization to non-logical constants An extended version of skolemization which matches eGTS is straightforwardly definable: not only existentially quantified variables and disjunctions, but now individual constants $a_{i}$ as well as relation symbols $R_{j}$ can be replaced by existentially quantified functions (for simplification I will no longer consider function symbols at the object language level.) In what follows we will consider a simple example - a precise definition is given in an appendix. Let us recall what are the first- and second-order Skolem forms $\mathbf{S k}[\varphi]$ and $\mathbf{2} \mathbf{S k}[\varphi]$ :

$$
\begin{aligned}
\varphi & =\forall x \exists y R_{4}\left(x, y, a_{2}\right) \\
\operatorname{Sk}[\varphi] & =\forall x R_{4}\left(x, \mathbf{f}(x), a_{2}\right) \\
\mathbf{2 S k}[\varphi] & =\exists f \forall x R_{4}\left(x, f(x), a_{2}\right)
\end{aligned}
$$

Let's now introduce some new notations. In $\mathbf{e S k}[\varphi]$ the non-logical constants are replaced by function symbols: one corresponding to a choice function $\mathbf{g}$ for the individual constant $a_{2}$, and another, $\mathbf{h}$, for the indicator function ${ }^{6}$ of the relation symbol $R_{4}$; since we want $\mathbf{e S k}[\varphi]$ to be equivalent to $\mathbf{S k}[\varphi]$, the newly introduced symbols are equated with their intended values: ${ }^{7}$

$$
\mathbf{e S k}[\varphi]=\forall x[\mathbf{h}(x, \mathbf{f}(x), \mathbf{g})=1] \wedge\left[\mathbf{g}=a_{2}\right] \wedge\left[\mathbf{h}=\mathbf{1}_{R_{4}}\right]
$$

Then in $\mathbf{2 e S k}[\varphi]$ the new function symbols are existentially quantified:

$$
\operatorname{2eSk}[\varphi]=\exists g \exists h \exists f\left[\forall x[h(x, f(x), g)=1] \wedge\left[g=a_{2}\right] \wedge\left[h=\mathbf{1}_{R_{4}}\right]\right]
$$

\footnotetext{
${ }^{6}$ Here again, one has to assume that the domain contains at least two elements, one of which is denoted by 1 . As was suggested by a referee, using relations symbols and predicate variables rather than indicator functions and function symbols would provide much more readable Skolem forms. However, the uniform use of (Skolem-like) functions at a metalinguistic level appears to be consistent with the fact that games are played with no higher-order entities. Incidentally, this uniform treatment would allow us to translate the resulting $\Sigma_{1}^{1}$ formulas into IF logic in the usual way.

${ }^{7}$ Both kinds of functions are linked to choice functions in the strict sense: the individual constant functions $\mathbf{g}_{a_{i}}$ could be defined relative to a domain $D$ s.t. $\mathbf{g}_{a_{i}}(D) \in D$; the indicator functions for relations $\mathbf{h}_{R_{j}}$ completely determine functions $\mathbf{h}_{R_{j}}^{\prime}$ s.t. $\mathbf{h}_{R_{j}}^{\prime}\left(D^{n}\right) \in D^{n}$.
} 
Assuming the standard semantics for second-order logic any first-order formula $\varphi=\Phi\left(R_{1}, \ldots, R_{k}, a_{1}, \ldots, a_{l}, x_{1}, \ldots, x_{m}\right)$ is equivalent to $2 \mathbf{e S k}[\varphi]$. (For more details, see Appendix B.)

Truth-conditions Prima facie the new game rules do not add any interesting moves to the plays since they say that a play should end in the same manner as was indicated in the original rule (R.At): the verifier wins if the atom is true, while the falsifier wins if it is false. And in fact the following equivalence is trivially inferred from the truth-definitions:

$$
\mathbf{M} \vDash_{\mathrm{eGTS}}^{+} \varphi \Longleftrightarrow \mathbf{M} \mathrm{F}_{\mathrm{GTS}}^{+} \varphi .
$$

However the specific benefits of GTS were not obvious either since it was shown to be equivalent to Tarski-like semantics. Regarding eGTS, relevant differences will appear at the level of the initial verifier's power, since mastering a winning strategy is now a more complex task than it was in standard GTS. The underlying intuition of atomic games is an extra requirement for $\exists$ loise: she must be able to determinate the semantic values of the expressions involved in the assertions she argues for. This is made clear if we are to describe her winning strategies:

A winning strategy for $\exists$ loise in some (unextended) GTS game $G(\varphi)$ is an array of Skolem functions related to each choice to be made by her through the game $\overline{\mathbf{f}_{\varphi}}=\left\langle\mathbf{f}_{\varphi}^{1}, \mathbf{f}_{\varphi}^{2}, \ldots \mathbf{f}_{\varphi}^{n}\right\rangle$; correspondingly, a winning strategy for $\exists$ loise in the eGTS game $e G(\varphi)$ is an array $e \overline{\mathbf{f}_{\varphi}}=\left\langle\mathbf{f}_{\varphi}^{1}, \mathbf{f}_{\varphi}^{2}, \ldots \mathbf{f}_{\varphi}^{n}\right.$, $\left.\left[\mathbf{f}_{\text {atom }}\right]\right\rangle$ where $\left[\mathbf{f}_{\text {atom }}\right]$ is the set of Skolem functions correlated with the individual constants and relation symbols occurring in $\varphi$ : $\left[\mathbf{f}_{\mathrm{atom}}\right]=$ $\left\{\mathbf{g}_{a_{1}}, \ldots, \mathbf{g}_{a_{k}}, \mathbf{h}_{R_{1}}, \ldots, \mathbf{h}_{R_{l}}\right\}$.

Considering formula $\varphi$ of example (5), its eGTS truth-conditions are given by (7) whereas the winning strategies for $\exists$ loise are provided by each interpretation of the function variables $\mathbf{f}, \mathbf{g}$ and $\mathbf{h}$ in (6). Of course, as long as the players of semantic games are ideal, infinitary and omniscient agents the requirement cannot trigger any fatal issue: the extensional constraint occurring onto the functions of $\left[\mathbf{f}_{\text {atom }}\right]$ is that their values coincide with those of the interpretation function $I$ of the structure. More accurately, each function $\mathbf{h}_{R_{i}} \in\left[\mathbf{f}_{\text {atom }}\right]$ must be extensionally equivalent to $\mathbf{1}_{R_{i}}$, so $\mathbf{h}_{R_{i}}$ is completely determined by $\left.I\right|_{\left\{R_{i}\right\}}$; and similarly each (constant) function $\mathbf{g}_{a_{j}} \in\left[\mathbf{f}_{\text {atom }}\right]$ must be completely determined by $\left.I\right|_{\left\{a_{j}\right\}}$. The union of the elements of $\left[\mathbf{f}_{\mathrm{atom}}\right]$ thus gives exactly the same values as the restriction of the interpretation function $I$ to the non-logical constants involved in $\varphi$.

The particular insight offered by Skolem functions will then be to provide a new point of view on the interpretation of a language. Whereas $I$ is a static 
mapping given once and for all, the Skolem functions of $\left[\mathbf{f}_{\text {atom }}\right]$ are expected to encode the devices used by the initial verifier to reach the semantic values of the language constants. The specific contribution of eGTS will thus be made apparent when some restrictions on the agents' powers are introduced. The situation is analogous to that of (unextended) GTS, which gives rise to a genuine account when imperfect information comes into play. 8,9

\section{An Account of Meaning and Interpretation Based on Game Strategies}

\subsection{Meaning: Implemented Skolem functions}

Using strategies to refine meaning It has been suggested by van Benthem [1] to use game-semantics in order to account for (hyper)fine-grained meanings: two logically equivalent sentences in FOL can diverge in their syntactic structure so that the corresponding evaluation games require different strategies. It would thus be possible to define the meaning of a sentence $\varphi$ not only as the set of possible worlds where $\varphi$ is true, but as the set of pairs $\langle\mathbf{M}, w s\rangle$ such that $\mathbf{M}$ is a possible world where $\varphi$ is true, and ws is the set of winning strategies for $\exists$ loise in $G(\varphi, \mathbf{M})$.

However, whereas the situation is fine with logical constants and unextended GTS, it is different with non-logical constants and eGTS atomic games: now, every component of the winning strategies should coincide with the interpretation function. Then if two predicates (or individual constants) are coextensive in every possible world, adding their eGTS correlated winning strategies cannot add any distinctive information. So no straightforward refinement of meanings can be expected from these strategies.

How to individuate Skolem functions? What is required is an intensional characterization of functions. This is the first issue eGTS has to face in order to avoid collapsing into standard GTS. Coextensive functions will be considered distinct whenever they are associated with different processes to determine their values. Of course, the identity conditions of

\footnotetext{
${ }^{8}$ Imperfect information semantic games provide a new logic which has been developed by Hintikka and Sandu since the 1980s, independence-friendly logic (IF logic for short). It is a slight extension of first-order logic equivalent to the $\Sigma_{1}^{1}$ fragment of second-order logic.

${ }^{9}$ Jackson [10] provides another extension of GTS to atomic formulas in the context of knowledge-base management: new game rules are introduced to check whether the atom in question is a proof-theoretic consequence of the knowledge base under consideration. The theory departs from the model-theoretic ground and admits of indeterminate formulas. Such an account can be seen as an implementation of non-omniscient players, where the initial verifier's knowledge is restricted to the content of the knowledge base.
} 
processes are worth defining, ${ }^{10}$ but what is required here is only the possibility of an intensional characterization of functions based on the idea of process, whatever it is.

The idea meets Moschovakis' [11] conception of Fregean sense as an algorithm, which is also elaborated by Muskens in [12]. What is specific here is the nature of the functions, which are winning strategies in games. However, we are not committed to sticking to the algorithm or program approach. Skolem functions are what is expected to be at least partially mastered by competent speakers and they can be realized by many distinct devices. We will be concerned with implemented Skolem functions in general - even though I might speak of 'algorithms' in a loose way.

Let us introduce a new symbol for extensional identity $-f \equiv g$ for $\forall \vec{x} f(\vec{x})=g(\vec{x})$ - and retain the identity symbol for intensional identity between functions, i.e. for identity between implemented functions. The truthconditions of a sentence $\varphi$ would be now expressed by the formula coming from $2 \operatorname{eSk}[\varphi]$ after a shift of the identity statements into coextensivity statements, for the Skolem functions correlated with constants $\left(\left[g_{i} \equiv a_{i}\right]\right)$ as well as for those linked to relations $\left(\left[h_{i} \equiv \mathbf{1}_{R_{i}}\right]\right)$. Such a requirement is weaker than that of identity of implemented functions.

eGTS Meaning Why not simply endorse Musken's theory and get rid of atomic games? But we would then lose the unifying framework of semantic games and of Skolem functions. Several NL semantic theories employ choice and Skolem functions to account for indefinites or anaphoric pronouns ([15], [20]). Schlenker [17] and Clark [3] even provide explicit connections between choice or Skolem functions uses in NL semantics and GTS. In fact the very idea was already developed by Hintikka with branching quantifiers. The proposal in this paper is then to extend the use of Skolem functions to NL semantics - and of the correlative GTS games - to the vocabulary, i.e. to names and predicates. It entails that Musken's intensionally characterized functions correlated with names and predicates are naturally interpreted as implemented strategies or Skolem functions.

Since in general linguistic expressions $E$ are correlated with many such implemented functions, I will denote by $\|E\|$ the set of implemented Skolem functions associated with $E$, and I will call it the eGTS meaning of E. For example, if there were two mechanisms (i.e., concrete devices) to identify dogs, the corresponding functions $\mathbf{h}_{1}$ and $\mathbf{h}_{2}$ would be elements of \|Dog\|. The concept of eGTS meaning so defined is model relative, and it will be used as implicitly relative to the actual world.

\footnotetext{
10 And this is not an easy task, see [2].
} 


\subsection{Interpretation: Implemented Dig functions}

"Strategic Meaning" As was said above, for any FO formula $\varphi$ there is an equivalence between $\mathbf{2} \mathbf{e S k}[\varphi]$ and $\mathbf{2 S \mathbf { k }}[\varphi]$ and this implies that no specific insight on the truth-conditions can be given by our new (atomic) games. A new perspective obtains if we examine closer what is expected from the initial verifier. Her winning strategies for molecular games are now enriched with functions for the atomic games involved. As was already noticed, there is no change of the conditions for her to have such strategies: there is a winning strategy for $\exists$ loise in the molecular game iff there is a winning strategy for her in the whole (including the atomic) game.

Atomic games are relevant insofar as one wants to know which particular functions are involved in a winning strategy. This is the case when bounded players are assumed to play the semantic games or, better, when some (human, concrete) agent is expected to know something about, or to use part of the winning strategy of one (abstract) player. Roughly said the issue is then no longer to know de dicto whether there is a winning strategy but to know de re which strategy will be a winning one.

Such knowledge is that of what Hintikka [6] labelled 'strategic meaning.' It is involved in semantic analysis when the 'abstract meaning' (i.e., the truth-condition) is no longer enough, such as in cases of ambiguity resolution, or in anaphora resolution. According to Hintikka, many features can play a role in the determination of the winning strategies: contextual data, background knowledge, syntactical clues, etc. Strategic meaning is a free extra offered by GTS, beyond the more usual truth-conditional content. It can sometimes be handled by speakers for the interpretation of particular occurrences of sentences in discourse or conversation. Hence strategic meaning can be considered as belonging to the pragmatic side of language. However, as Hintikka deservedly insists, strategic meaning is just a by-product of a semantic theory and must be considered as such.

Adding one dimension: interpretation The idea of strategic meaning suggests making a move from meaning constitution to linguistic interpretation (or understanding), not only at the level of complex sentences but also at the level of atomic ones. Meaning constitution is accounted for by semantic games played by the abstract players in a model-theoretic structure. Linguistic interpretation is another type of game: it is concerned with real, limited speakers in concrete contexts. This will lead us to a relativization of the notions of eGTS to contexts and to approximations of Skolem functions. As requiring a total grasp of meaning (hence of extensions) by competent speakers is not realistic, it is necessary to modify the theory developed up to now, and regiment the following three ideas about linguistic knowledge: (i) It is contextual knowledge: there is an elimination of algorithms in the 
general case; (ii) It is partial knowledge: there is a specific elimination of algorithms relatively to the agent; (iii) It is approximate knowledge: linguistic knowledge might involve functions which are not always winning strategies, i.e. not always Skolem functions.

(i) Contextual knowledge. The ability to determine the extension of expressions in order to understand an utterance is relative to its context. Both the way the context is modeled, and the question why what in the context that counts as important does the job in the interpretation process, are issues that go beyond the purpose of this section. I will rather claim in a very general manner that contexts are expected to play a specific role regarding Skolem functions: contexts should restrict the class of the relevant, hence available, Skolem functions.

Indeed, my claim highlights a role of contexts which is very often emphasized in the literature, namely quantifier domain restriction (see [18] for a general overview.) Semantic theories more specifically employing Skolem functions usually also take contextual restrictions into account. This is the case of Hintikka's [6] conception of strategic meaning according to which the context restricts the set of available winning strategies. Von Heusinger [19] provides a uniform semantic representation for pronouns: "Pronouns are understood as referring to the most salient individual in the context so far, and are represented as indexed epsilon terms that are interpreted by a choice function [which] reconstructs the salience structure of the context." Therefore, the role played by the context in the setting of the relevant choice functions appears to be a pervarsive feature of NL semantic theories involving such functions.

(ii) Partial knowledge. There are in general connections linking the speaker to the individuals and properties she refers to in her utterance. In eGTS, these connections correspond to the Skolem functions of [ $\left.\mathbf{f}_{\mathrm{atom}}\right]$. If such connections are individuated at the level of their implementations, they may become dependent of the context: some algorithm or other is available in such or such a context, but not in another. For instance, as the speaker is an element of the context, complexity considerations can occur and imply some restriction on the available devices. More generally speaking the location of the utterance can play an important role on the selection of a device to determine the extension of an expression. One can ponder over the calculation of the extension of "Water" in a desert $\mathbf{h}_{\text {water }}^{c_{1}}$ which may diverge from the corresponding calculation in a kitchen $\mathbf{h}_{\text {water }}^{c_{2}}$.

For the framework of eGTS, what all this means is that relative to a given context $c$, there are contextual restrictions $\|E\|^{c}$ on the set of (implemented) winning strategies of the initial verifier $\|E\|$ generally available for a given concrete speaker. Some of these restrictions are due to the location of the speaker, but not necessarily all of them. For some utterances of a sentence, 
there might be no available Skolem function at all even though the sentence is true in the model - i.e. even though there actually are such functions for the initial verifier of the corresponding semantic game.

(iii) Approximate knowledge. Speakers are far from omniscient. In his wellknown paper [13] Putnam argued that a speaker who could not discriminate between "Elms" and "Beeches" might nonetheless be considered a competent English speaker. A fixed amount of approximation is indeed acceptable according to the linguistic community - the exact amount being of course conventionally determined. For instance, a speaker who could not discriminate between "Snowball" and "Tiger" would not be considered competent.

So competent speakers can employ implemented functions (i.e., concrete devices) to determine the extension of predicates that do not necessarily coincide with the interpretation function of the underlying model. Consequently such functions are not necessarily Skolem functions of eGTS, even though some of them can be. I will call these functions Dig functions. They are those strategies used by concrete speakers if they were to calculate the extensions of the predicates and names they employ, sometimes winning, sometimes losing.

eGTS stereotype The set of Dig functions eventually associated with an expression $E$ by a competent speaker $i$ can thus strongly diverge from the eGTS meaning $\|E\|$ : it can come from both a contraction of the set of Skolem functions, and an enlargement to many losing strategies. Following Putnam's ideas let us call this set the stereotype of $E$ for $i$ and denote it by $+E t_{i}$. As for the corresponding set of Skolem functions, we must use the restriction $+E t_{i}^{c}$ to model the stereotype of an agent $i$ in a context $c$. Stereotypes are specific to the agents' idiolects. Some of them are acceptable, others not, depending on social conventions. What counts as a socially acceptable stereotype is a matter of empirical research.

We finally reached a two-dimensional account of "meaning" (in a broad sense), eGTS meaning and eGTS stereotype, with no resort to possibleworld semantics. Intensionality intervenes at the level of the individuation of functions, and a second refinement comes from the interpretation level. In the next section, I will give a few applications of the two notions.

\section{Application and Philosophical Consequences}

\subsection{Contrasting meaning and interpretation}

Meaning It is now time to consider how eGTS handles concrete cases. Let us consider a simple discourse: 


\section{A man walks. He kicks Nicolas}

Its analysis according to GTS is quite standard (see [8], [16], [3]); according to eGTS, the game should go deeper. It is roughly played as follows:

The whole game $e G$ is played relative to a given model $\mathbf{M}=\langle D, I\rangle$. It is cut into two subgames, one for each sentence - i.e., $e G=e G\left(S_{1}, \mathbf{M}\right)$; $e G\left(S_{2}, \mathbf{M}\right)$, where $S_{1}$ stands for "A man walks", and $S_{2}$ for "He kicks Nicolas." In $e G\left(S_{1}, \mathbf{M}\right) \exists$ loise chooses an individual in $D$ for the initial indefinite "A", say $b$, and puts it in the choice set CS; then the game goes on as $e G(b$ is a man and $b$ walks, $\mathbf{M})$. $\forall$ belard chooses a conjunct, say $b$ is a man, and the game continues as $e G(b$ is a man, $\mathbf{M})$. We have now reached an atomic formula: if it is true, i.e., if $\exists$ loise actually chose a man with $b$, then $\forall$ belard cannot do anything clever ${ }^{11}$ but choose the predicate "man", and pick out an arbitrary individual, say $b^{\prime}$, from the domain $D$; $\exists$ loise answers yes if $b^{\prime}$ is a man, and no if it isn't a task she can successfully achieve since she is omniscient. Moving to $e G\left(S_{2}, \mathbf{M}\right)$, $\exists$ loise chooses an individual in the choice set $C S$ to process the anaphoric pronoun "He". So she picks out $b$, and the game goes on as $e G(b$ kicks Nicolas, $\mathbf{M})$. This is an atomic formula. If it is true, i.e. if the individual chosen by $\exists$ loise in the previous subgame actually kicks Nicolas, then $\forall$ belard chooses one of its two non-logical constants, say Nicolas. $\exists$ loise must then find the bearer of the proper name in $D$, a job she easily does as an omniscient player.

Assuming that the above discourse is true, there is a winning strategy for $\exists$ loise in $e G$. What would such a strategy look like? It is a sequence of (sets of) implemented Skolem functions:

$$
\left\langle\mathbf{f}_{1},\left\{\mathbf{h}_{1}, \mathbf{h}_{2}\right\}, \mathbf{f}_{2},\left\{\mathbf{h}_{3}, \mathbf{g}_{1}\right\}\right\rangle
$$

where: $\mathbf{f}_{1} \equiv \mathbf{f}_{2}$ pick out a man walking and kicking Nicolas, $\mathbf{h}_{1} \in \|$ Man\|, $\mathbf{h}_{2} \in \|$ Walk $\left\|, \mathbf{h}_{3} \in\right\| \mathrm{Kick} \|$, and $\mathbf{g}_{1} \in \|$ Nicolas $\|$. Hence, $\exists$ loise's winning strategies directly rely on the eGTS-meanings of the predicates and proper name. The target array for $\exists$ loise actually is

$$
\left\langle\mathbf{f}_{1},\{\|\operatorname{Man}\|, \| \text { Walk } \|\}, \mathbf{f}_{2},\{\| \text { Kick }\|,\| \text { Nicolas } \|\}\right\rangle .
$$

This array provides a basis to construe the eGTS-meaning of the whole discourse. Such an analysis requires adding specific rules to combine elementary meanings (those occurring in the array) in a more subtle, syntax-sentitive way than it is in a mere sequence. But that would lead us beyond the scope of the present paper.

\footnotetext{
11 Strictly speaking, $\forall$ belard could also choose the individual constant $b$ and lose immediately.
} 
Interpretation While shifting from meaning to interpretation, contexts of utterance, limitations of the agents and approximation enter the scene. As an immediate consequence, one has to address the issue of what minimal amount of linguistic knowledge is expected to be mastered by any competent speaker.

Let us consider the interpretation of the above discourse by an agent $i$ hearing it: differently from $\exists$ loise, $i$ need not master any winning strategy of the corresponding eGTS game. Her target array looks like

$$
\left\langle\mathbf{f}_{1}^{i},\left\{\text { Mant }_{i}, \text { +Walkt }_{i}\right\}, \mathbf{f}_{2}^{i},\left\{\text { Kickt }_{i}, \text { NNicolast }_{i}\right\}\right\rangle .
$$

where $\exists$ loise's implemented functions $\mathbf{f}_{1}$ and $\mathbf{f}_{2}$ have been replaced by a couple of others, $\mathbf{f}_{1}^{i}$ and $\mathbf{f}_{2}^{i}$ respectively, and the elementary meanings have been changed into the corresponding stereotypes for agent $i$. Roughly said, $i$ must know that the value produced by $\mathbf{f}_{2}^{i}$ should be the same as that selected by $\mathbf{f}_{1}^{i}$, i.e $\mathbf{f}_{2}^{i} \equiv \mathbf{f}_{1}^{i}$, which corresponds to the understanding of the anaphoric pronoun - but she need not know anything about the function $\mathbf{f}_{1}^{i}$ itself, except that it should take its values among male human beings. Furthermore, a competent hearer must have some socially acceptable stereotype +Mant ${ }_{i}$, but nothing is required about "Nicolas", i.e. †Nicolast $_{i}$ can be empty.

It means that the minimal knowledge required of a competent speaker can vary between logical categories. Of course, an agent can have more than such a minimal knowledge and use strategic meaning to interpret a discourse. In the example, the agent can know the functions' value (i.e., who the man kicking Nicolas is), and her stereotype Nicolast $_{i}$ can be a proper part of the corresponding GTS-meaning (i.e., it can involve implemented functions that actually pick out the bearer of the name.)

In the remainder of the subsection I will check three categories, namely proper names, relations, and definite descriptions, and examine what corresponding knowledge competent speakers are supposed to possess.

Names An implemented Skolem function correlated to a name $a$ is a mechanism $\mathbf{g}_{a}$ which produces the referent of the name. It represents any mode of identification of its bearer. However, an assertion making use of a proper name can refer to the bearer of the name even though the speaker who utters it does not master any of the corresponding Skolem functions in $\|a\|$. For a competent speaker $i$ to successfully assert: "He kicks Nicolas" - and to successfully refer to Nicolas - it is only required that the truth condition of the statement be realized, namely that the ordered pair made of the individual picked out by he, and of the bearer of the name I(Nicolas), whoever they are, be in the extension $I$ (Kick) of the kicking relation, whatever this extension is. If the speaker has no idea who is the bearer of the name, +Nicolast ${ }_{i}$ is 
simply empty. ${ }^{12}$ Of course, the knowledge of the bearer of a name would increase one's understanding of the sentence where the name occurs, but it is not required.

The semantics based on Skolem and Dig functions is perfectly compatible with the Kripkean theory of direct reference for proper names - as well as for natural kind predicates and other relation symbols. Whereas the interpretation function $I$ intervenes at the level of meaning constitution, implemented Skolem and Dig functions play an epistemic role: they encode the mode of recognition of the independently given extension of expressions. Hence different speakers knowing the individual that is the bearer of a proper name usually do not share the same Dig functions. The contrast is exactly the one which holds between abstract meaning and strategic meaning.

Predicates (and other relation symbols) In the actual world, one can have two or more functions to determine the extension of "Elm". This is the main reason why stereotypes were defined as sets of Dig functions. For instance one function will be based on the agent's knowledge and educated ability to recognize species of trees; another one will consist in asking to an expert what this extension is. For sure, these functions are distinct at an implementation level even though they provide the same values.

The functions involved in the stereotype are clearly distinct from intensions. The latter are usually modelled as functions from possible worlds to extensions. By contrast, Dig functions are defined in one (actual) world, with no a priori idea about their applicability in other possible worlds. Nevertheless, Dig functions can model concepts expressed by predicates. Coextensive predicates, such as Creature owning a heart $(H x)$ and Creature owning a kidney $(K x)$, will obviously give rise to two distinct sets of implemented Skolem functions (or eGTS meanings) $\|H\|$ and $\|K\|$. Each function is then expected to encode an operating way to produce the extension, i.e. to determine whether any given object belongs to it or not. They can naturally be conceived of as encoding the correlated concepts of Owning a heart and Owning a kidney, which play an essential role in the speaker's ability to produce the extension of the predicates. And of course, this distinction between two coextensive predicates is made with no resort to any possible-world based device.

As was said before, a competent speaker is expected to possess a stereotype composed of Dig functions which yield a socially acceptable approximation of the extension. For instance a speaker $i$ who cannot discriminate between "Elms" and "Beeches", i.e. such that $+E \operatorname{lmt}_{i}=\mathrm{HBeech}_{i}$ can nonetheless be considered a competent English speaker although of course,

\footnotetext{
12 If the reader doesn't share my intuition, successful reference is more obvious in cases such as the speaker's assertion: "I do not know who Nicolas is".
} 
†Elm† $\dagger_{\text {Expert }} \cap+$ Beech $\dagger_{\text {Expert }}=\varnothing$. By contrast, a speaker $j$ who could not discriminate between "Elms" and "Tigers", would not be considered a competent speaker, a minimal requirement being that: $+\mathrm{Elm}_{j} \cap+\mathrm{Tiger}_{j}=\varnothing \cdot{ }^{13}$ Beyond the minimal requirement of the possession of at least one Dig function, the understanding of a predicate will be more accute if the speaker has several Dig functions, if many of them are Skolem functions, if she can use them successfully in many counterfactual sitations, and so forth.

Definite descriptions With definite descriptions we are led back to a case similar to non-extended GTS since quantifiers occur in their formalization. However, the interaction between quantifiers and atomic games deserves careful examination.

Considering a sentence such as "The 2009 French president is bald", the description $2 x P x$ ("The 2009 French president") is paraphrased by an existential statement and the whole sentence is formalized as follows:

$$
\varphi: \exists x(P x \wedge \forall y(P y \rightarrow y=x) \wedge B x)
$$

Here the GTS truth-conditions rely on the existence of a winning strategy for the initial verifier, i.e. of a constant Skolem function $\mathbf{f}_{x}=a$ such that $\exists$ loise yields a winning strategy in any of the three games correlated to the conjuncts:

$$
P a \wedge \forall y(P y \rightarrow y=a) \wedge B a
$$

$\exists$ loise has thus to hold two Skolem functions associated with the predicates and coinciding with the interpretation function, $\mathbf{h}_{P}$ and $\mathbf{h}_{B}$, so that she is able to win the atomic games $P a$ and $B a$, as well as the game associated with $\forall y(P y \rightarrow y=a) .{ }^{14}$ Once again, the existence of a winning strategy for the initial verifier of a semantic game - abstract meaning - is independent from the knowledge of any strategy by the speaker - strategic meaning.

A complete knowledge of the strategy corresponding to the definite description in the aforementioned example involves knowledge of the two functions $\mathbf{f}_{x}(=a)$ and $\mathbf{h}_{P}$. However a competent speaker is not expected to master both functions: having a function that enables one to approximately

\footnotetext{
13 To be more precise in the writing down of the constraints, we should relativize the stereotypes to usual contexts, in order to avoid situations where, e.g., an elm and a tiger were both boiled, ground, then mixed with flour and black ink, so that the agent can no longer distinguish them. This relativization is here left implicit.

14 This subformula is equivalent to $\forall y(\neg P y \vee y=a)$, so that $\exists$ loise has a winning strategy if, for any value chosen for $y$ by $\forall$ belard, she can select one of the disjuncts, i.e. either deny $P$ of this value or identify it to $a$; it is of course assumed that the function $\mathbf{h}_{P}$ does not change during the game. Finally, a winning strategy for the initial verifier in the whole game associated with $\varphi$ is a tuple where $\mathbf{h}_{P}$ occurs twice - one time for each occurrence of $P$ in $\varphi: \overline{\mathbf{f}_{\varphi}}=\left\langle\mathbf{f}_{x},\left\langle\left\{\mathbf{h}_{P}\right\},\left\langle\mathbf{f}_{\vee},\left\{\mathbf{h}_{P}\right\}\right\rangle,\left\{\mathbf{h}_{B}\right\}\right\rangle\right\rangle$.
} 
determine the denotation of the description $\left(\mathbf{h}_{P}\right)$ - i.e. possessing an acceptable stereotype $+\mathrm{PH}_{i}$ - is enough; knowing who the individual fitting the description $\left(\mathbf{f}_{x}\right)$ is increases the understanding of the sentence, although not absolutely essential. The case is partly similar to that of proper names. As a definite description involves a combination of predicates and quantified variables, the minimal requirements for a competent speaker follow those of its components: no strategic meaning is required regarding quantifiers, whereas a conventionally determined stereotype is expected to be used in the case of predicates.

\subsection{Philosophical puzzles}

With eGTS meanings and stereotypes, we possess two levers to get solutions to the classical semantic puzzles about informative identities, conceivability of impossibilities, and so forth. In this last subsection, I will briefly survey some of these puzzles.

Informative identities They can be carried using eGTS meanings. For instance, $\|$ Hesperus $\|\neq\|$ Phosphorus $\|$ since the implemented Skolem functions corresponding to each proper name are not the same, even though they are extensionally equivalent: if $\mathbf{g}_{H} \in \|$ Hesperus $\|$ and $\mathbf{g}_{P} \in \|$ Phosphorus $\|$, then $\mathbf{g}_{H} \equiv \mathbf{g}_{P}$ but $\mathbf{g}_{H} \neq \mathbf{g}_{P}$.

Skolem and Dig functions can be understood as carrying a part of the Fregean notion of sense: they represent the devices employed respectively by $\exists$ loise and by ordinary speakers to find the extension. Of course, Dig functions are not Frege's senses: they do not determine the extension per se, at the semantic or constitutive level.

The informativeness of the identity is due to the fact that speakers are not expected to know anything about the bearers of the names they employ. Learning an identity is consequently learning a fact about Dig functions and, more basically, about the bearers of the names: learning that Hesperus is identical with Phosphorus leads an agent $i$ to correlate functions $\mathbf{g} \in$ tHesperust $_{i}$ to functions $\mathbf{g}^{\prime} \in$ tPhosphorust $_{i}: \mathbf{g} \equiv \mathbf{g}^{\prime}$. By contrast, stating that Hesperus is identical with Hesperus doesn't supply any new information.

Rigid designation Going into modal matters and following Kripke, we can consider the proper names "Hesperus" and "Phosphorus" as rigid designators, i.e. as names referring to the same entity in every possible world. How are we then to account for the conceivability of impossible situations where the two names do not refer to the same entity? This puzzle gave rise 
to several strategies, like two-dimensional semantics or rescourse to impossible worlds. On the present account, the structure of possible worlds itself is left intact with no ad hoc supplementary component, and Skolem (or dig) functions suffice to account for conceivable impossible situations.

Let us consider the two implemented functions mentioned above, $\mathbf{g}_{H}$ and $\mathbf{g}_{P}$. As Skolem functions, they can occur in winning strategies for $\exists$ loise in semantic games $G(\varphi, \mathbf{M})$, where $\varphi$ is replaced by sentences involving the proper names "Hesperus" and "Phosphorus", and $\mathbf{M}$ is a first-order model corresponding to the actual world. Recall that such functions can diverge in another model $\mathbf{N}$ corresponding to another possible world, i.e. $\mathbf{g}_{H} \not \equiv \mathbf{g}_{P}$ in $\mathbf{N}$, even though the proper names still refer to the same individual in $\mathbf{N}$.

In this respect, Skolem functions behave like Hintikka's world-lines [5] or like individual concepts: they link individuals from world to world according to specific viewpoints, and independently from the very nature of the individuals at stake. ${ }^{15}$ This is made possible by the general fact that a winning strategy in a game $G(\psi, \mathbf{M})$ need not be winning in the game $G\left(\psi, \mathbf{M}^{\prime}\right)$, i.e. in the game correlated to the same sentence but played in another model.

Empty names Stereotypes and Dig functions provide a nice solution to the usual puzzles since there is now a way to make a difference between two empty names (e.g. Shrek and Pinocchio), and a means to designate entities in other possible worlds. What is required is only to allow Dig functions to be partial, i.e. to be possibly undefined in some possible worlds. Alternatively, it can be postulated that the gaps are filled in with a null individual. There is no prima facie objection to the consideration of two distinct Dig functions, $\mathbf{g}_{p}$ for Pinocchio and $\mathbf{g}_{s}$ for Shrek, such that they be empty in some worlds and their values coincide in no world in which they are defined: such functions thus account for the meaningfulness of fictional discourse in a natural way.

Synonymy cases The well-known cases of the ignorance of synonymous predicates and, as a consequence, of the equivalence of sentences involving them, are handled in the present framework in a way similar to that of coreferent proper names: even though their extensions coincide in every possible world, the eGTS meanings for e.g. "Ophthalmologist" $(O)$ and "Eye doctor" $(E)$, which are sets of implemented Skolem functions, do not: $\|O\| \neq$ $\|E\|$; and the corresponding stereotypes of course do not either: tot $\neq$ †E†. This is enough to account for possible distinctive attitudes towards sentences whose equivalence is due to synonymy. Ralph can believe that George is an eye doctor and simultaneously believe that George is not an ophthalmologist,

\footnotetext{
15 This means that in a standard Kripkean structure, the accessibility relation between possible worlds is doubled over by world-lines between entities. However, Hintikka does not agree with such a combination of his own ideas with that of rigid designation.
} 
without being inconsistent: Ralph's first belief involves a device - i.e., an implemented function - from $\mathrm{HE}_{R}$, whilst the second one involves one from ${ }^{+0} t_{R}$. Hence the contents of the attitudes are not the same.

Twin-Earth The present conception enables one to deal with cases like Putnam's Twin-Earth [13]. E-Oscar and TE-Oscar, the microphysical twins living on Earth and Twin-Earth, are supposed to share the same (narrow) mental state as they think about "Water", even though E-Oscar refers to $\mathrm{H}_{2} \mathrm{O}$ whereas TE-Oscar refers to XYZ. It can be assumed that the twins share their stereotypes: twatert $_{\mathrm{E}-0 \mathrm{scar}}=$ twater $_{\mathrm{TE}-0 \mathrm{scar}}$.

The Dig functions of E-Oscar enable him to determine the extension of "Water" on Earth. They are not necessarily Skolem functions, i.e. functions picking out water in every context, but they do so in terrestrial contexts: it is only their restriction to terrestrial contexts which coincides with that of Skolem functions, i.e. twatert ${ }_{\mathrm{E}-0 \text { scar }}^{\text {Earth }} \subseteq \|$ water $\|^{\text {Earth }}$. Using the same stereotype on Twin-Earth, TE-Oscar will select another type of extension, namely XYZ. So the extensions depend on the environment - it is the assumption - whereas the stereotype is shared by the twins.

\section{Conclusion}

I have shown that a slight extension of GTS could yield interesting results for the theory of meaning. This can be done provided that one does not stick to the usual static and extensional conception of functions as mappings but adopts some fine-grained criterion of individuation of functions - namely, one must move at the implementation level. Here, concrete devices must be introduced in order to account for interpretation by limited agents to be contrasted with the semantic games played by unlimited players. Hence after meaning constitution through eGTS comes the step of linguistic understanding, where context, approximation, and other epistemic issues enter the stage.

The conception put forward in this paper can be viewed as an elaboration on Putnam's ideas about meaning. The key is in the strict separation between meaning constitution and language understanding. Putnam appended the notion of stereotype to that of meaning. In our framework, there is a shift from sets of Skolem functions to sets of Dig functions: limited agents are fallible - as they interpret language, they do not automatically grasp functions that yield the appropriate extensions. This is why the devices are not Fregean senses, but rather make up Putnamian stereotypes.

The resulting conception is a non-modal account of meaning and interpretation: Skolem and Dig functions are defined in one single world. The 
fine-grained individuation of such functions certainly require intensionality, but it is limited to the metalinguistic level. Moreover, the whole conception is based on an informal interpretation of functions as concrete devices which is a long shot from usual possible-world semantics: here competent speakers are not expected to grasp functions defined on sets of possible worlds, let alone two-dimensional functions.

\section{Appendices}

\section{A eGTS rules}

In eGTS, every FO sentence $\varphi$ evaluated relative to a structure $\mathbf{M}=\langle D, I\rangle$ is associated to a game $e G(\varphi, \mathbf{M})$. This new game is identical to the game $G(\varphi, \mathbf{M})$ played according to the standard GTS rules when it is a molecular game, i.e. when $\varphi$ is a complex formula. If $\varphi$ is an atomic formula or an identity, one reaches an atomic game. The original game rule for atomic sentences in GTS, (R.At), is replaced by the following specific four rules:

- (R.At*). In the atomic game e $G(\alpha, \mathbf{M})$, if either $\exists$ loise is the current verifier and $\alpha$ is false in $\mathbf{M}$, or she is the current falsifier and $\alpha$ is true, then $\forall$ belard wins and $\exists$ loise loses; else there are two cases: (i) $\alpha$ is of the form $R t_{1} \ldots t_{n}$, $R$ being a $n$-ary relation symbol and the $t_{i}$ s being terms: $\forall$ belard picks out an index $i \in\{0,1, \ldots, n\}$; if $i=0$ then the rest of the game is as in $e G(R, \mathbf{M})$, else it is as in $e G\left(t_{i}, \mathbf{M}\right)$. (ii) $\alpha$ is of the form $\left(t_{1}=t_{2}\right)$, the $t_{i}$ s being terms: $\forall$ belard picks out an index $i \in\{1,2\}$; the rest of the game is as in $e G\left(t_{i}, \mathbf{M}\right)$.

- (R.Rel). In the relation game $e G(R, \mathbf{M})$, where $R$ is a $n$-ary relation symbol, $\forall$ belard chooses a $n$-tuple $\left\langle d_{1}, \ldots, d_{n}\right\rangle \in D^{n}$, and $\exists$ loise answers yes or no. If she answers yes and $\left\langle d_{1}, \ldots, d_{n}\right\rangle \in I(R)$, or she answers no and $\left\langle d_{1}, \ldots, d_{n}\right\rangle \notin$ $I(R)$ then $\exists$ loise wins and $\forall$ belard loses; else $\forall$ belard wins and $\exists$ loise loses.

- (R.Term). In the term game e $G(t, \mathbf{M})$, where $t$ is a term, one of the following three cases occurs:

(i) $t$ is a new constant previously introduced through the play i.e. not occurring in the original formula; then $\exists$ loise wins and $\forall$ belard loses;

(ii) $t$ is an individual constant; then $\exists$ loise chooses an object $d \in D$; if $d=I(t)$ then $\exists$ loise wins and $\forall$ belard loses; if $d \neq I(t)$ vice versa;

(iii) $t$ is a complex term involving a $n$-ary function symbol: $t=f\left(t_{1}, \ldots, t_{n}\right)$; then $\forall$ belard picks out an index $i \in\{0,1, \ldots, n\}$. If $i>0$ then then rest of the game is as in $e G\left(t_{i}, \mathbf{M}\right)$, and if $i=0$ it is as in $e G(f, \mathbf{M})$.

- (R.Fun). In the function game $e G(f, \mathbf{M}), f$ being a $n$-ary function symbol, $\forall$ belard chooses a $n$-tuple $\left\langle d_{1}, \ldots, d_{n}\right\rangle \in D^{n}$, then $\exists$ loise chooses an object $d \in D$. If $d=I(f)\left(d_{1}, \ldots, d_{n}\right)$ then $\exists$ loise wins and $\forall$ belard loses; else vice versa. 


\section{B Second-order Skolem forms}

There is a simple device to yield a result equivalent to extended GTS with no resort to (sub)atomic games, but only to skolemization. In what follows we will assume that the models under consideration contain at least two distinct elements, and that second-order formulas get a standard (full) semantic interpretation. Let:

$$
\Phi\left(a_{1}, \ldots, a_{k}, R_{1}, \ldots, R_{l}, x_{1}, \ldots, x_{m}\right)
$$

be a first-order sentence, where $a_{1}, \ldots, a_{k}$ are the $k$ individual constant symbols, $R_{1}, \ldots, R_{l}$ the $l$ relation symbols, and $x_{1}, \ldots, x_{m}$ the $m$ variables occurring inside $\Phi$. Being first-order $\Phi$ can be put into prenex normal form:

$$
Q_{1} x_{1} \ldots Q_{m} x_{m} \Phi^{\otimes}\left(a_{1}, \ldots, a_{k}, R_{1}, \ldots, R_{l}, x_{1}, \ldots, x_{m}\right)
$$

with $Q_{i} \in\{\exists, \forall\} . \Phi$ can also be skolemized, and is equivalent to:

$$
\begin{aligned}
\operatorname{2Sk}[\Phi]=\exists f_{1} \ldots \exists f_{n} \forall t_{1} \ldots \forall t_{p} \\
\qquad \Phi^{\circ}\left(a_{1}, \ldots, a_{k}, R_{1}, \ldots, R_{l}, f_{1}\left(\overrightarrow{t_{1}}\right), \ldots, f_{n}\left(\overrightarrow{t_{n}}\right), t_{1}, \ldots, t_{p}\right)
\end{aligned}
$$

where $\left\{t_{1}, \ldots, t_{p}\right\} \subseteq\left\{x_{1}, \ldots, x_{m}\right\}$ is the subset of the universally quantified variables of $\Phi^{\otimes}, \vec{t}_{i} \subseteq\left\{t_{1}, \ldots, t_{p}\right\}$ is the set of the universally quantified variables of $\Phi^{\otimes}$ on which the existentially quantified variable replaced by $f_{i}$ depends, and $\Phi^{\circ}$ results from $\Phi^{\otimes}$ by a mere permutation of its arguments so that the Skolem functions appear first.

Now we can replace the relation and individual constant symbols in $\Phi$ by secondorder quantified variables. So $\Phi$ is equivalent to the following formula:

$$
\begin{aligned}
\exists g_{1} \ldots \exists g_{k} \exists X_{1} \ldots \exists X_{l}\left[\Phi\left(g_{1}, \ldots, g_{k}, X_{1}, \ldots, X_{l}, x_{1}, \ldots, x_{m}\right)\right. \\
\left.\wedge\left[g_{1}=a_{1}\right] \wedge \ldots \wedge\left[g_{k}=a_{k}\right] \wedge\left[X_{1}=R_{1}\right] \wedge \ldots \wedge\left[X_{l}=R_{l}\right]\right]
\end{aligned}
$$

The same existential generalization can be done within $\mathbf{2 S k}[\Phi]$, reaching the following formula:

$$
\begin{aligned}
\exists g_{1} \ldots \exists g_{k} \exists & X_{1} \ldots \exists X_{l} \exists f_{1} \ldots \exists f_{n} \forall t_{1} \ldots \forall t_{p} \\
& {\left[\Phi^{\circ}\left(g_{1}, \ldots, g_{k}, X_{1}, \ldots, X_{l}, f_{1}\left(\overrightarrow{t_{1}}\right), \ldots, f_{n}\left(\overrightarrow{t_{n}}\right), t_{1}, \ldots, t_{p}\right)\right.} \\
& \left.\wedge\left[g_{1}=a_{1}\right] \wedge \ldots \wedge\left[g_{k}=a_{k}\right] \wedge\left[X_{1}=R_{1}\right] \wedge \ldots \wedge\left[X_{l}=R_{l}\right]\right]
\end{aligned}
$$

Furthermore, let us change each relation variable $X_{i}$ into a corresponding (indicator) function variable $h_{i}$ and correlatively modify $\Phi^{\circ}$ into $\Phi^{\star}$ - so that each token of $X_{i} t_{1} \ldots t_{n_{i}}$ be replaced by one of $h_{i}\left(t_{1} \ldots t_{n_{i}}\right)=1$. Hence $\Phi$ is equivalent to its extended second-order Skolem form:

$$
\begin{aligned}
\operatorname{2eSk}[\Phi]_{1}= & \exists g_{1} \ldots \exists g_{k} \exists h_{1} \ldots \exists h_{l} \exists f_{1} \ldots \exists f_{n} \forall t_{1} \ldots \forall t_{p} \\
& {\left[\Phi^{\star}\left(g_{1}, \ldots, g_{k}, h_{1}, \ldots, h_{l}, f_{1}\left(\overrightarrow{t_{1}}\right), \ldots, f_{n}\left(\overrightarrow{t_{n}}\right), t_{1}, \ldots, t_{p}\right)\right.} \\
& \left.\wedge\left[g_{1}=a_{1}\right] \wedge \ldots \wedge\left[g_{k}=a_{k}\right] \wedge\left[h_{1}=\mathbf{1}_{R_{1}}\right] \wedge \ldots \wedge\left[h_{l}=\mathbf{1}_{R_{l}}\right]\right]
\end{aligned}
$$

A similar first-order skolemization is then given by:

$$
\begin{aligned}
\mathbf{e S k}[\Phi]=\forall & t_{1} \ldots \forall t_{p}\left[\Phi^{\star}\left(\mathbf{g}_{1}, \ldots, \mathbf{g}_{k}, \mathbf{h}_{1}, \ldots, \mathbf{h}_{l}, \mathbf{f}_{1}\left(\overrightarrow{t_{1}}\right), \ldots, \mathbf{f}_{n}\left(\overrightarrow{t_{n}}\right), t_{1}, \ldots, t_{p}\right)\right. \\
& \left.\wedge\left[\mathbf{g}_{1}=a_{1}\right] \wedge \ldots \wedge\left[\mathbf{g}_{k}=a_{k}\right] \wedge\left[\mathbf{h}_{1}=\mathbf{1}_{R_{1}}\right] \wedge \ldots \wedge\left[\mathbf{h}_{l}=\mathbf{1}_{R_{l}}\right]\right]
\end{aligned}
$$


Another second-order Skolem form, equivalent to (16), obtains if we restore the original constants in $\Phi^{\star}$ :

$$
\begin{aligned}
2 \mathbf{e S k}[\Phi]_{2}= & \exists g_{1} \ldots \exists g_{k} \exists h_{1} \ldots \exists h_{l} \exists f_{1} \ldots \exists f_{n} \forall t_{1} \ldots \forall t_{p} \\
& {\left[\Phi^{\circ}\left(a_{1}, \ldots, a_{k}, R_{1}, \ldots, R_{l}, f_{1}\left(\overrightarrow{t_{1}}\right), \ldots, f_{n}\left(\overrightarrow{t_{n}}\right), t_{1}, \ldots, t_{p}\right)\right.} \\
& \left.\wedge\left[g_{1}=a_{1}\right] \wedge \ldots \wedge g_{k}=a_{k}\right] \\
& \left.\wedge\left[h_{1}=\mathbf{1}_{R_{1}}\right] \wedge \ldots \wedge\left[h_{l}=\mathbf{1}_{R_{l}}\right]\right]
\end{aligned}
$$

which can be written in a condensed form as:

$$
\begin{aligned}
\mathbf{2 e S k}[\Phi]_{2}=\exists g_{1} \ldots \exists g_{k} \exists h_{1} \ldots \exists h_{l} \\
\quad\left[\mathbf{2} \mathbf{S k}[\Phi] \wedge\left[g_{1}=a_{1}\right] \wedge \ldots \wedge\left[g_{k}=a_{k}\right] \wedge\left[h_{1}=\mathbf{1}_{R_{1}}\right] \wedge \ldots \wedge\left[h_{l}=\mathbf{1}_{R_{l}}\right]\right]
\end{aligned}
$$

Acknowledgements. Previous and partial versions of this work were presented on several occasions during the past years - notably at APLI 2006 (Rijeka, Croatia), at JSM 2007 (Paris, France), and at the Universidade Nova de Lisboa (2008). I wish to thank Denis Bonnay, Paul Egré, Bertram Kienzle, Paul Gochet, Helge Rückert, Anna Sierszulska, and Tero Tulenheimo for their comments on earlier versions of this paper and for many fruitful discussions. All errors remain mine.

\section{References}

[1] van Benthem, J., 'The Epistemic Logic of IF Games', in R. E. Auxier \& L. E. Hahn, eds., The Philosophy of Jakko Hintikka, The Library of Living Philosophers Volume XXX, Open Court, 481-512, 2006.

[2] Andreas Blass, A., Dershowitz, N., \& Gurevich Y., 'When Are Two Algorithms the Same?', The Bulletin of Symbolic Logic, 15, 2, 145-168, 2009.

[3] Clark, R., 'Games, Quantifiers and Pronouns', in A.-V. Pietarinen (ed.), Game Theory and Linguistic Meaning, Amsterdam, Elsevier: 139-159, 2007.

[4] Fodor, Jerry A., 1987: Psychosemantics: The Problem of Meaning in the Philosophy of Mind, Cambridge M.A., MIT Press.

[5] Hintikka, J., 'Semantics for Propositional Attitudes', in J. W. Davis, D. J. Hockney \& W. K. Wilson (eds.), Philosophical Logic, D. Reidel Publishing Company, Dordrecht, 21-45, 1969.

[6] Hintikka, J., 'Language Understanding and Strategic Meaning', Synthese 73: 497-529, 1987.

[7] Hintikka, J., 'A Second Generation Epistemic Logic and Its General Significance', in V.F. Hendricks, S.A. Pedersen, and K.F. Jørgensen (eds.), Knowledge Contributors, Dordrecht, Kluwer Academic Publishers: 33-55, 2003.

[8] Hintikka, J., \& Kulas, J., Anaphora and Definite Descriptions: Two Applications of Game-Theoretical Semantics, Dordrecht, D. Reidel, 1985. 
[9] Hintikka, J., \& Sandu, G., 'Game-Theoretical Semantics', in J. van Benthem \& A. ter Meulen (eds.), Handbook of Logic and Language, Elsevier, Amsterdam, 361-410, 1997.

[10] Jackson, P., 'On Game-Theoretic Interactions with First-Order Knowledge Bases', in P. Smets et al. (eds.) Non-Standard Logics for Automated Reasoning, London, Academic Press: 27-54, 1988.

[11] Moschovakis, Y., 'Sense and Denotation as Algorithm and Value', Logic Colloquium '90 (Helsinki 1990), Vol. 2 of Lecture Notes in Logic, Springer, Berlin: 210-249, 1994.

[12] Muskens, R., 'Sense and the Computation of Reference', Linguistics and Philosophy 28: 473-504, 2005.

[13] Putnam, H., 'The Meaning of 'Meaning", in K. Gunderson (ed.), Language, Mind and Knowledge, Minneapolis (MN), University of Minnesota Press, 1975.

[14] Rebuschi, M., 'Contenu étroit, mécanisme et fonctions de choix', Philosophie 100: 77-94, 2008 .

[15] Reinhart, T., 'Quantifier Scope. How labor is Divided Between QR and Choice Functions', Linguistics and Philosophy 20: 335-297, 1997.

[16] Sandu, G., On the Theory of Anaphora: Dynamic Predicate Logic vs. GameTheoretical Semantics, Linguistics and Philosophy 20, 147-174, 1997.

[17] Schlenker, Ph., 'Scopal Independence: A Note on Branching \& IslandEscaping Readings of Indefinites \& Disjunctions', Journal of Semantics, 23 (3): 281-314, 2006.

[18] Stanley, J. and Z.G. Szabó: 'On Quantifier Domain Restriction', Mind 86 Language 15 (2\&3): 219-261, 2000.

[19] von Heusinger, K., 'Reference and Representation of Pronouns', in H.J. Simon and H. Wiese (eds.), Pronouns - Representation and Grammar, Amsterdam, Philadelphia, Benjamin: 109-135, 2002.

[20] Winter, Y., 'Functional Quantification', Research on Language and Computation 2: 331-363, 2004.

Manuel Rebuschi

L.H.S.P. - Archives H. Poincaré (UMR 7117)

Université Nancy 2

91, avenue de la Libération - BP 454

F-54001 NANCY Cedex (France)

Manuel.Rebuschi@univ-nancy2.fr 